University of Nebraska - Lincoln

DigitalCommons@University of Nebraska - Lincoln

April 1995

\title{
Role of Specific Response Elements of the $c$-fos Promoter and Involvement of Intermediate Transcription Factor(s) in the Induction of Sertoli Cell Differentiation (Transferrin Promoter Activation) by the Testicular Paracrine Factor PModS*
}

\author{
Patricia D. Whaley \\ University of California at San Francisco \\ Jaideep Chaudhary \\ University of California at San Francisco \\ Andrea S. Cupp \\ University of Nebraska-Lincoln, acupp2@unl.edu \\ Michael K. Skinner \\ University of California at San Francisco
}

Follow this and additional works at: https://digitalcommons.unl.edu/animalscifacpub

Part of the Animal Sciences Commons

Whaley, Patricia D.; Chaudhary, Jaideep; Cupp, Andrea S.; and Skinner, Michael K., "Role of Specific Response Elements of the $c$-fos Promoter and Involvement of Intermediate Transcription Factor(s) in the Induction of Sertoli Cell Differentiation (Transferrin Promoter Activation) by the Testicular Paracrine Factor PModS*" (1995). Faculty Papers and Publications in Animal Science. 160.

https://digitalcommons.unl.edu/animalscifacpub/160

This Article is brought to you for free and open access by the Animal Science Department at DigitalCommons@University of Nebraska - Lincoln. It has been accepted for inclusion in Faculty Papers and Publications in Animal Science by an authorized administrator of DigitalCommons@University of Nebraska - Lincoln. 


\title{
Role of Specific Response Elements of the c-fos Promoter and Involvement of Intermediate Transcription Factor(s) in the Induction of Sertoli Cell Differentiation (Transferrin Promoter Activation) by the Testicular Paracrine Factor PModS*
}

\author{
PATRICIA D. WHALEY $\dagger$, JAIDEEP CHAUDHARY, ANDREA CUPP, AND \\ MICHAEL K. SKINNER \\ Reproductive Endocrinology Center, University of California, San Francisco, California 94143-0556
}

\begin{abstract}
A mesenchymal-epithelial cell interaction exists in the testis between the Sertoli cells that form the seminiferous tubule and the mesenchymal-derived peritubular myoid cells that surround the tubule. Analysis of the mesenchymal-epithelial interactions between these cells revealed the local production of a mesenchymal factor, PModS. PModS modulates the differentiated functions of Sertoli cells in vitro, including stimulation of the iron-binding protein transferrin (Tf). Previous results have indicated that PModS-induced Tf gene expression involves the activation of immediate early genes. One of the immediate early genes was identified as c-fos. The importance of c-fos was demonstrated in the current study when a c-fos antisense oligonucleotide was found to inhibit the ability of PModS to induce the expression of a Tf promoter-chloramphenicol acetyltransferase (CAT) construct. The regulation of c-fos by PModS was investigated with various CAT constructs containing segments of the c-fos promoter, such as the serum response element (SRE), sis-inducible element (SIE), cAMP response element (CRE), and phorbol ester/IPA response element (TRE), transfected into cultured Sertoli cells. PModS has no effect on cAMP response element-CAT or TRE-CAT, suggesting that PModS does not act through stimulation of $\mathrm{cAMP}$ and protein kinase $\mathrm{C}$ pathways. PModS was found to activate the c-fos SRE-CAT construct and the SIE-CAT construct. A construct containing both SIE and SRE was stimulated to the same degree as either element alone. Gel mobility shift assays using nuclear extracts from PModSstimulated Sertoli cells and a radiolabcled SRE oligonucleotide resulted in retarded mobility of a DNA-protein complex. A gel shift with a SRE oligonucleotide containing an ETS domain resulted in a unique shift only detected in PModS-stimulated cells. PModS also promoted a gel shift with the SIE that is adjacent to the SRE on the c-fos promoter. The data imply that PModS can activate the c-fos promoter
\end{abstract}

through the SRE and SIE. PModS caused a labeled activating protein 1 (AP1) oligonucleotide to form a DNA-protein complex, indicating activation of the c-fos gene and binding of the c-fos/jun complex. To study the downstream regulation of Sertoli cell differentiation, Tf gene expression was examined. CAT constructs containing deletion mutants of a 3-kilobase $(\mathrm{kb})$ mouse Tf promoter were used. When transfected into Sertoli cells the 581-base pair Tf minimal promoter had only a slight response to PModS, but was activated by FSH. The 2.6-kb Tf promoter construct responded to PModS. This response was greater than that observed with the 1.6- or $3-\mathrm{kb}$ Tf promoter constructs. These results suggest that an upstream enhancer located in the Tf promoter between -2.6 and $-1.6 \mathrm{~kb}$ is responsive to PModS. Gel retardation assays with two restriction fragments, designated $\mathrm{SE} 1$ and SE2, located at -2.4 and $-1.9 \mathrm{~kb}$, respectively, on the $\mathrm{Tf}$ promoter showed a retarded complex with PModS-stimulated Sertoli cell nuclear extracts. No other restriction enzyme fragment of the Tf promoter was found to cause a gel shift. These SE1 and SE2 domains are in a region with apparent enhancer activity and were found not to contain a c-fos/jun AP1-binding site. An immunoblot with c-fos antibodies of the SE1 and SE2 gel shift indicated the absence of c-fos in the DNA-protein complex. Therefore, PModS-responsive cis-elements on the Tf promoter were identified (i.e. SE1 and SE2) that do not appear to involve a direct activation by c-fos at AP1, but involve an intermediate c-fos-regulated transcription factor(s). The findings suggest that PModS acts through activation of the SRE and SIE to induce the immediate early gene, c-fos, which then influences an intermediate transcription factor(s) that regulates downstream Sertoli cell differentiated functions, such as Tf expression. These studies have initiated an investigation of the transcriptional regulation of Sertoli cell differentiation. (Endocrinology 136: 3046-3053, 1995)
A MESENCHYMAL -epithelial cell interaction exists in the testis between the Sertoli cells that form the seminiferous tubule and the mesenchymal-derived peritubular myoid cells that surround the tubule. Analysis of mesenchymal-epithelial interactions between these cells revealed

Received December 15, 1994

Address all correspondence and requests for reprints to: Dr. Michael K. Skinner, Reproductive Endocrinology Center, University of California, San Francisco, California 94143-0556.

* This work was supported by the University of California-San Francisco Reproductive Endocrinology Center (HD-11979), NIH Grant (HD20583; to M.K.S.), and a Rockefeller Postdoctoral Fellowship (to J.C.).

+ Current address: Clonetech Laboratories, Inc., Palo Alto, California 94303. the local production of a mesenchymal factor, termed PModS $(1,2)$. The testicular paracrine factor, PModS, is produced by the peritubular myoid cells under androgen control and modulates Sertoli cell differentiated functions in vitro $(1,2)$. $\Lambda$ mong these differentiated functions is the synthesis of the iron-binding protein, transferrin (Tf) $(3,4)$. Tf expression has been used as a marker of Sertoli cell differentiation during pubertal development (4). To investigate the pharmacology of PModS, several common signal transduction pathways were analyzed. PModS was found to have no effect on cAMP levels (4) or on calcium mobilization or phosphoinositide metabolism (5). Although PModS was found to elevate cGMP, cGMP does not mediate the actions of PModS (5). 
Treatment of Sertoli cells with the tyrosine phosphorylation inhibitor, genistein, suppresses PModS-stimulated Tf production (5). Therefore, the actions of PModS on Sertoli cells appear to be in part through an activation of tyrosine kinase(s). Regulation of Sertoli cell differentiation by PModS on a molecular level has also been investigated. An inhibitor of translation, cycloheximide, was used to determine whether PModS could directly stimulate Tf messenger RNA (mRNA) expression or whether intermediate protein synthesis was involved. Cycloheximide inhibited PModS-induced Tf mRNA synthesis, indicating that activation of immediate early genes is required for stimulation of $\mathrm{Tf}$ expression (6). Northern analysis of potential immediate early genes demonstrated that PModS stimulates c-fos mRNA synthesis, but has no effect on two other nuclear proteins c-jun and C/EBP. The protein c-fos is a protooncogene that is transiently stimulated by a variety of mitogens and differentiation factors (7). Time-course data show that c-fos mRNA levels increase within minutes after PModS treatment and subsequently decline to basal levels after $2 \mathrm{~h}$. In contrast, PModS-induced increases in Tf mRNA do not occur until $2 \mathrm{~h}$, then peak at approximately $12 \mathrm{~h}$, and are maintained for up to 5 days in culture. Treatment of Sertoli cells with a c-fos antisense oligonucleotide partially suppresses PModS-enhanced Tf protein production (6). These data indicate that $c-f o s$ is involved as an immediate early gene in response to PModS. The current study was designed to analyze PModS activation of the c-fos promoter and relate this to the previous data on signal transduction and Sertoli cell differentiation. Activation of the Tf promoter was investigated as a downstream event involved in Sertoli cell differentiation.

\section{Materials and Methods}

\section{Cell preparation and culture}

Sertoli cells were isolated from the testis of 20-day-old rats by sequential enzymatic digestion (8), with a modified procedure described by Tung et al. (9). Decapsulated testicular fragments were digested first with trypsin ( $1.5 \mathrm{mg} / \mathrm{ml}$; Gibco-BRL, Gaithersburg, MD) to remove the interstitial cells and then with collagenase $(1 \mathrm{mg} / \mathrm{ml}$ type I; Sigma Chemical Co., St. Louis, MO) and hyaluronidase (1 mg/ml; Sigma). Sertoli cells were then plated under serum-free conditions in 24-well Falcon plates at $1 \times 10^{6}$ cells $/$ well. Cells were maintained in a $5 \% \mathrm{CO}_{2}$ atmosphere in Ham's F-12 medium (Gibco-BRL) at $32 \mathrm{C}$. Sertoli cell cultures were treated as described in Results, after $48 \mathrm{~h}$ of culture and transfection. Cultures were treated with FSH $(100 \mathrm{ng} / \mathrm{ml}$; ovine FSH-16, National Pituitary Agency), $(\mathrm{Bu})_{2}$ CAMP $(1 \mathrm{~mm}), 10 \%$ calf serum, or greater than maximally effective concentrations of PModS(S300) $(25 \mu \mathrm{g} / \mathrm{ml})$ or PModS(C4) $(25 \mathrm{ng} / \mathrm{ml})$.

Peritubular cells were obtained from the collagenase digestion supernatant, as described by Skinner et al. (2), after the tubule segments had sedimented. Peritubular cells were plated in Ham's F-12 medium containing $10 \%$ calf serum and grown to confluence. Cells were then subcultured and plated at $25 \%$ confluence. When subcultured cells were confluent, they were washed with serum-free medium. The cells were subsequently cultured in serum-free medium for up to 4 weeks with 48- to 72-h medium collections.

Freshly collected serum-free conditioned medium from the peritubular cells was treated with phenylmethylsulfonylfluoride ( $25 \mu \mathrm{M}$ final concentration) and benzamidine ( $0.1 \mathrm{~mm}$ final concentration) and centrifuged at $1000 \times \mathrm{g}$ for $15 \mathrm{~min}$ at $4 \mathrm{C}$ to remove cell debris. When necessary, medium was stored at $-20 \mathrm{C}$. Conditioned medium was concentrated 100-fold by ultrafiltration with an Amicon system (Amicon Corp., Lexington, MA) and a $3000 \mathrm{~mol}$ wt exclusion limit membrane.

\section{PModS preparation}

PModS was obtained from concentrated peritubular cell-conditioned medium, as previously described (2). An ammonium sulfate precipitate of concentrated conditioned medium was applied to a size-exclusion Sephacryl S300 column (Pharmacia, South San Francisco, CA). The active peak of the S300, determined by bioassay of Sertoli cell Tf production, was collected and applied to a chromatofocusing column (Pharmacia). The $\mathrm{pH}$ 5.7-6.8 pool was applied to a $1 \times 15-\mathrm{cm}$ heparinSepharose affinity column. Eluted proteins were applied to two successive C4 reverse phase columns (Vydac, Hesperia, CA) and eluted with a linear gradient from 25-60\% acetonitrile. The partially purified PModS S300 pool, termed PModS(S300), and the more highly purified PModS(C4) fraction, termed PModS(C4), were stored at $-70 \mathrm{C}$ before use in the presence of $1 \mathrm{mg} / \mathrm{ml}$ BSA.

\section{Reporter gene constructs}

c-Fos promoter. The chloramphenicol acetyltransferase (CAT) reporter plasmids (PBL-CAT2) with the thymidine kinase minimal promoter (10) containing either the CAMP response element (CRE) [120-base pair (bp) $5^{\prime}$-flanking sequence from the transcriptional start site of the human c-fos promoter with a 5'-TGACGTTT-3' sequence at $-60 \mathrm{bpl}$, TPA/activating protein 1 (AP1) response element (TRE; $5^{\prime}$-TGCGTCA-3'), or the serum response element (SRE; 5'-CAGGATGTCCATATTAGGACATC-3') sequences of the c-fos promoter were generated; the entire c-fos promoter was generously provided by Dr. Jeff Holt (Vanderbilt University, Nashville, TN). The sis-inducible element (SIF)-CAT reporter plasmid was constructed by cloning a synthetic 15 -bp oligonucleotide (CATTTCCCGTAAATC) (11) with HindIII and SphI sites on either ends into PBL CAT2 plasmid. The SIE/SRE construct was prepared by cloning the 15-bp SIE oligo into HindIII/SphI sites of the SRE-CAT plasmid.

Tf promoter. The CAT reporter plasmid containing $-581 \mathrm{bp}$ (PUC8 CAT) and the human GH reporter plasmid containing -3.0 -kilobase $(\mathrm{kb})$ sequences of the mouse Tf (mTf) promoter (12) were generously provided by Dr. G. Stanley McKnight (University of Washington, Seattle, WA). The CAT reporter plasmids containing $-3.0-\mathrm{kb} \mathrm{mTf}$ promoter and its deletions were constructed as follows. 1) The $3.0-\mathrm{kb}$ mTf CAT construct was made by ligating a $-3.0-\mathrm{kb}$ BamHI-BamHI digest to the BamHI site in PGL2-CAT plasmid (Promega). 2) The 2-kb upstream HindIII-HindIII fragment in the 3-kb mTf promoter was ligated in the HindIII site of -581 -bp mTf-CAT to obtain the $2.6-\mathrm{kb}$ mTf-CAT plasmid. 3) The -1.6-kb mTf CAT plasmid was derived from the $-3.0-\mathrm{kb} \mathrm{mTf}$ CAT (no. 1 above) by digesting out the upstream $-1.4-\mathrm{kb}$ Pst I fragment. 4) To prepare the upstream 1-kb mTf-CAT (1-kb Tf-Tk-CAT), the 3-kb mTf plasmid was digested with HindIII-PstI. The $1-\mathrm{kb}$ fragment $(1.6-2.6$ $\mathrm{kb}$ ) was then cloned into a PBL-CAT2 plasmid.

Constructs for each of the plasmids that did not contain the specific response element or promoter fragment (i.e. promoterless plasmid) were also generated. In all cases, experiments were performed to examine the actions of various treatments on these promoterless plasmids. As discussed in Results, these promoterless plasmids generally did not respond to the treatments. If a small response was observed, the data are shown in the figures or stated in Results.

\section{Transfection}

Sertoli cells, cultured for $\mathbf{4 8} \mathrm{h}$, were transfected with a reporter gene construct by the calcium phosphate method coupled with hyperosmotic shock (10\% glycerol), as previously described (13). Cells were treated 2 $h$ after transfection, and unless otherwise stated, CAT activity was determined after $48 \mathrm{~h}$ in culture.

\section{CAT assay}

Assay of CAT activity was performed as follows. Medium was removed from the wells, and the cells were washed once with PBS. One hundred microliters of $1 \times$ cell lysis buffer (Promega Corp., Mad- 
ison, WI) were added to each well and incubated for $15 \mathrm{~min}$ at room temperature. The wells were then scraped, and the buffer was collected in $1.5-\mathrm{ml}$ microfuge tubes. Tubes were heated to $65 \mathrm{C}$ for $10 \mathrm{~min}$ to inactivate endogenous acetylases and then spun at $12,000 \times \mathrm{g}$ for $10 \mathrm{~min}$ at $4 \mathrm{C}$ to remove cell debris. An aliquot of cell extract $(54 \mu \mathrm{l})$ was mixed with $65 \mu \mathrm{l} 0.25 \mathrm{M}$ Tris (pH 8.0), $25 \mu \mathrm{g} n$-butyryl coenzyme A $(5 \mathrm{mg} / \mathrm{ml}$; Sigma), and $0.1 \mu \mathrm{Ci}\left[{ }^{14} \mathrm{Clchloramphenicol}(0.1 \mu \mathrm{Ci} / \mu \mathrm{l}\right.$; ICN, Costa Mesa, CA) and incubated overnight at $37 \mathrm{C}$. The mixture was extracted once with $300 \mu \mathrm{l}$ xylene and back-extracted with $100 \mu \mathrm{l} 0.25 \mathrm{M}$ Tris (pH 8.0 ). A $200-\mathrm{ml}$ aliquot of the organic phase was counted in a scintillation counter to determine the relative amount of CAT activity.

\section{Gel mobility shift assay}

Gel shift assays were performed with nuclear extracts of isolated Sertoli cells. The Sertoli cells were isolated as described above and cultured in 137-mm petri dishes (Falcon Plastics, Oxnard, CA). The cells were treated after $48 \mathrm{~h}$ in culture either FSH, PModS(S300), or $10 \%$ calf serum. After $72 \mathrm{~h}$, the cells were scraped off the petri dishes and washed once with PBS. The nuclear extracts of these cells were then prepared as described by Guillou et al. (14). The probes used in gel retardation assays were the SRE (5'-GGATGTCCATATTAGGACACATCTG-3') and the ETS-SRE element (SRE with 5'-CAGGAT sequence) of the c-fos promoter; the SIE (5'-CATTTCCCGTAAATC-3') located 25 bp upstream of the c-fos SRE (15), and AP1 dimer (5'-TTAGTCATGAGTCA-3'). The complementary oligonucleotides were 5 -end labeled with $\left[\gamma^{32} \mathrm{P}\right] \mathrm{ATP}$ $(150 \mu \mathrm{Ci} / \mu \mathrm{l}$; New England Nuclear Corp., Boston, MA) using polynucleotide kinase, annealed, and subsequently purified on a $12 \%$ polyacrylamide gel.

Restriction fragments of the mTf promoter between -581 bp and -3 $\mathrm{kb}$ were obtained with a series of enzymes, including PvuII, NarI, HpaI, and PstI. Fifteen approximately 200-bp fragments were isolated, dephosphorylated, and end labeled with $\left[\gamma^{-32} \mathrm{P}\right] \mathrm{ATP}$ using polynucleotide kinase. Of these fragments, two gave a positive gel shift. The restriction fragments SE1 $(200 \mathrm{bp})$ and SE2 $(180 \mathrm{bp})$ were located at -2.4 and -1.9 $\mathrm{kb}$, respectively, on the $3-\mathrm{kb} \mathrm{mTf} 5^{\prime}$-flanking region. These two Tf promoter fragments (i.e. SE1 and SE2) were routinely isolated, dephosphorylated, and end labeled with $\left[\gamma^{-}{ }^{32} \mathrm{P}\right] \mathrm{ATP}$ for gel shift analysis.

The gel retardation assay used was a modification of the protocol described by Garner and Rezvin (15). The final reaction volume of $20 \mu \mathrm{l}$ contained $0.5 \mathrm{ng} 5^{\prime}$-end ${ }^{32} \mathrm{P}$-labeled double stranded probe, $100 \mathrm{ng}$ sonicated salmon sperm DNA, $2 \mu \mathrm{g}$ DNA duplex poly(dI-dC) (U.S. Biochemical Corp., Cleveland, $\mathrm{OH}$ ), $20 \mu \mathrm{g}$ BSA, 20 mм HEPES ( $\mathrm{pH}$ 8.0), 4 mм Tris (pH 7.9), $50 \mathrm{~mm} \mathrm{KCl}, 600 \mu \mathrm{M}$ EDTA (pH 8.0), EGTA (pH 8.0), $500 \mu \mathrm{M}$ dithiothreitol, and $5 \mu \mathrm{g}$ Sertoli cell nuclear proteins. After incubation at room temperature for $20 \mathrm{~min}, 5 \mu \mathrm{l}$ of the reaction were electrophoretically separated on a $5 \%$ polyacrylamide gel. The gel was dried and autoradiographed. For the competition experiments, a 500fold molar excess of unlabeled oligonucleotide or restriction fragment was added to the binding reaction.

\section{Antisense procedure}

A c-fos antisense oligonucleotide, 3'-TACTACGGTGCA-5', was prepared and used, as previously described $(16,17)$. A c-fos sense oligonucleotide was prepared for use as a negative control. Transfected Sertoli cells were treated with either the antisense or sense oligomer (4 $\mu \mathrm{M})$ starting $8 \mathrm{~h}$ after transfection and retreated every $8 \mathrm{~h}$ for a total of $48 \mathrm{~h}$ before harvest of the cells for CAT assay.

\section{Immunoblot procedure}

A gel mobility shift assay was electrophoretically transferred to a polyvinylidone difluoride membrane (Immobilon Millipore, South San Francisco, CA) by electrophoresis in Tris-glycine buffer containing 20\% methanol. The blot was then blocked with $3 \%$ nonfat milk [dissolved in

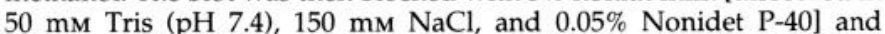
incubated with a 1:5000 dilution of antibody to c-fos (Santa Cruz Biotechnology, Santa Cruz, CA) for $2 \mathrm{~h}$. After three washes (15 min each), the blot was hybridized with a secondary antibody (1:3000 dilution; directed against rabbit immunoglobulin $\mathrm{G}$ ) conjugated to horseradish peroxidase for $1 \mathrm{~h}$ at room temperature. After five washes under the conditions described above, detection of the immune complex was performed using the chemiluminescent ECL method (Amersham Corp., Arlington Heights, IL).

\section{Statistical analysis}

Each data point was converted to a percentage of the control value, and the mean and SEM from multiple experiments were determined, as indicated in the figure legends. Data were analyzed by analysis of variance, using the SPSS Statistical Package (SPSS, Chicago, IL).

\section{Results}

To confirm that c-fos is involved at a molecular level in PModS stimulation of Tf, a CAT reporter gene construct containing $-2.6 \mathrm{~kb}$ of the Tf gene promoter was transiently transfected into cultured Sertoli cells. Sertoli cells were isolated from 20-day-old rat testis and cultured under serumfree conditions unless otherwise stated. After transfection, the cells were left untreated (control) or were treated with FSH, a partially purified PModS preparation from a sizeexclusion column termed PModS(S300), a combination of PModS(S300) and the antisense c-fos oligonucleotide (S300+ ANTI), or a combination of PModS(S300) and the sense c-fos oligonucleotide (S300+SENSE) for $48 \mathrm{~h}$ before analysis of the cell extracts for CAT activity. FSH, S300, and S300+SENSE activated the Tf CAT construct (Fig. 1). A control promoterless plasmid (pCAT-basic) was slightly stimulated by PModS(S300) treatment. Treatment of the transfected cells with the antisense oligonucleotide inhibited PModS(S300)-induced CAT activity (Fig. 1). These data confirm that c-fos is involved in PModS-stimulated Tf gene expression.

\section{c-Fos promoter analysis}

To determine how PModS activates c-fos gene expression, $\mathrm{CAT}$ reporter gene constructs containing different regulatory

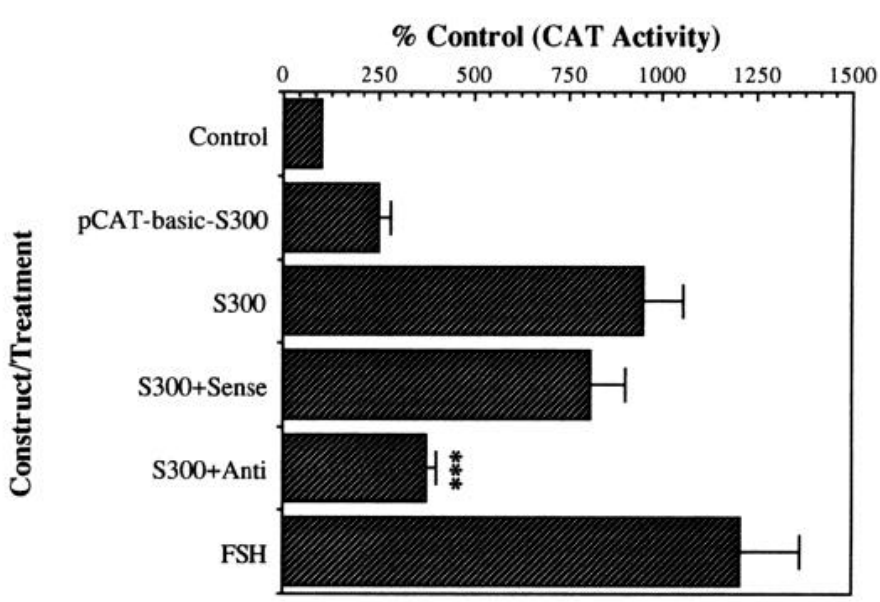

FIG. 1. Regulation of the 2.6-kb Tf promoter-CAT construct in Sertoli cells cultured in the absence (control; C) or presence of $\mathrm{FSH}$, PModS(S300), PModS(S300) and c-fos antisense oligonucleotide (S300+ANTI), and PModS(S300) and c-fos sense oligonucleotide (S300+ SENSE). The actions of PModS(S300) on a control pCAT-basic plasmid are also presented. Data are expressed as a percentage of the control and are presented as the mean \pm SEM from three different experiments performed in replicate. ${ }^{* * *}$, Statistical difference from S300 or S300+SENSE $(P<0.01)$. 
elements of the c-fos promoter were generated, including the CRE, TRE, SIE, and SRE. A schematic representation of the locations of these elements within the c-fos promotor is shown in Fig. 2A. These constructs were transiently transfected into cultured Sertoli cells. After transfection, the cells were left untreated (control) or were treated with FSH, 10\% calf serum, $(\mathrm{Bu})_{2} \mathrm{CAMP}$, PModS(S300), or more highly purified PModS preparations from the $\mathrm{C} 4$ reverse phase column termed PModS(C4) for $48 \mathrm{~h}$. The CRE of the c-fos promoter was activated by both $\mathrm{FSH}$ and $(\mathrm{Bu})_{2}$ CAMP $400 \%$ above the control level $(P<0.05)$, which demonstrated that the reporter construct can be activated (data not shown). Treatment with PModS(S300) did not increase CRE-CAT activity above the control level (Fig. 2B). Treatment of the c-fos TRE-transfected cells with FSH resulted in a $300 \%$ stimulation above the control level $(P<0.05)$, which demonstrated that this reporter construct can be activated (data not shown). Treatment of the transfected Sertoli cells with PModS(S300) does not stimulate the TRE construct (Fig. 2B). A SRE construct was used that included the 5'-flanking 5-bp ETS domain ETS-SRE sequence of the c-fos promoter. Activation of the c-fos SRE construct was accomplished by treatment with $10 \%$ calf serum as a positive control to demonstrate that the reporter construct can be activated (data not shown). Both PModS(S300) and PModS(C4) activated the SRE construct (Fig. 2B). The SIE construct was also stimulated by PModS(S300) and PModS(C4) (Fig. 2B). Interestingly, a construct containing both the SIE and SRE was stimulated to the

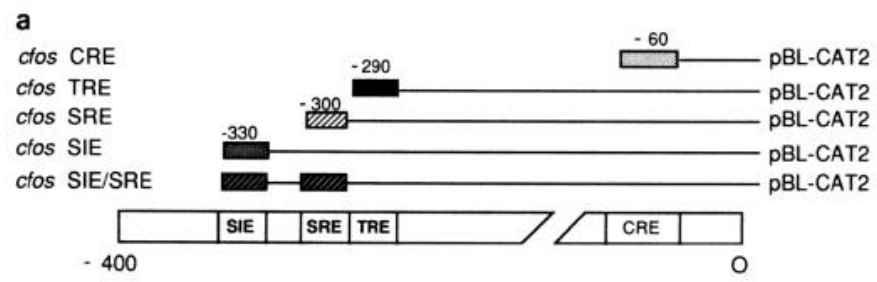

b

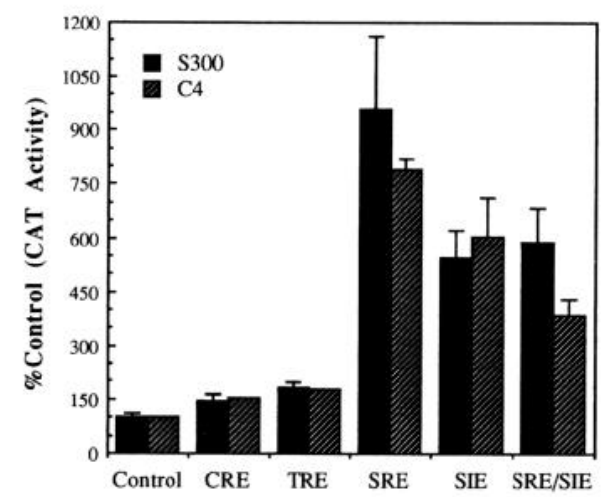

FIG. 2. a, Schematic representation of the c-fos promoter and the locations of the CRE, SRE, SIE, and TRE in promotor-CAT constructs. The designation for the promoter constructs is listed at the left, and that for the plasmid used at the right. b, Regulation of the c-fos promoter constructs (CRE, TRE, SIE, and SRE) in Sertoli cells cultured in the absence (control) or presence of PModS(S300) (ש) and PModS $(\mathrm{C} 4)(\mathbb{Z})$. Data are expressed as a percentage of the control and are presented as the mean \pm SEM from six different experiments performed in replicate. same or lesser degree as that containing either element alone. The entire 400-bp c-fos promoter construct was stimulated approximately $900 \%$ over the control level by PModS(S300) (data not shown). The control plasmids not containing a promoter were not influenced by any of the treatments (data not shown). Purified PModS had a similar action as the partially purified PModS(S300). This indicates that stimulation of c-fos expression by PModS is in part mediated through activation of the SRE and SIE.

To extend these observations, gel retardation/mobility shift assays were performed. Nuclear extracts were obtained from Sertoli cells cultured in the absence (control) or presence of PModS(S300) or $10 \%$ ( $\mathrm{vol} / \mathrm{vol})$ calf serum as a positive control. A gel shift with an oligonucleotide to Oct-1 was used to check the quality of the nuclear extracts (Fig. 3). All extracts resulted in a similar level of Oct- 1 gel shift. To confirm that c-fos was induced, a gel shift with an AP1 oligonucleotide was performed, because the c-fos-jun complex binds to the AP1 site. Incubation of the labeled AP1 oligonucleotide with nuclear extracts of Sertoli cells treated with PModS(S300) or serum resulted in the formation of a DNA-protein complex with retarded mobility (i.e. gel shift). The observed gel shift was specific for AP1, because it could be effectively competed by unlabeled AP1 (Fig. 3). This confirms the induction of c-fos by both PModS(S300) and serum.

Activation of SRE of the c-fos promoter was also investigated with a gel mobility shift assay using a SRE oligonucleotide (Fig. 4A). PModS(S300) induced a gel shift that was competed with excess unlabeled SRE. Serum was used as a positive control and also promoted a gel shift. To assess whether PModS may promote binding of a ternary complex, an ETS-SRE sequence was used in a gel shift. The ETS domain is a 5'-CAGGAT flanking sequence of the SRE that is needed for the binding of ETS oncogene-like proteins and is required for the formation of a ternary complex with the serum response factor (SRF) that binds to the SRE. PModS(S300) promoted a distinct shift with the ETS-SRE that was not present in control nontreated cells or in serum-treated cells (Fig. 4A). PModS(S300) promoted two distinct gel shifts, whereas serum promoted a diffuse shift below that of PModS(S300). These gel shifts were displaced with excess unlabeled oligonucleotide (data not shown). Therefore, PModS(S300) induced protein binding to the SRE and ETS-SRE oligonucleotides. An alternate response element, located $25 \mathrm{bp}$

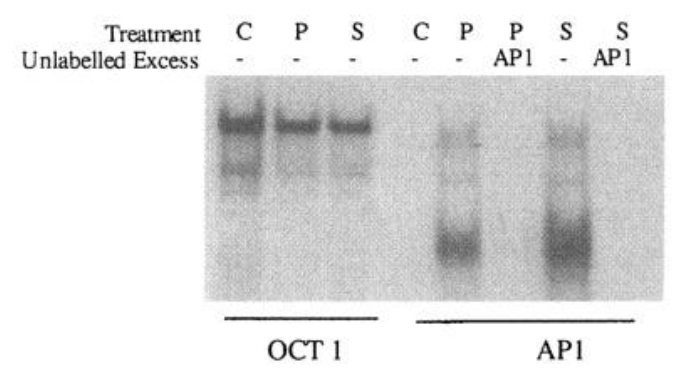

FIG. 3. Gel mobility shift assay with ${ }^{32} \mathrm{P}$-radiolabeled Oct-1 and AP1 oligonucleotides. Nuclear extracts from Sertoli cells cultured in the absence $(\mathrm{C})$ or presence of $\mathrm{PModS}(\mathrm{S} 300)(\mathrm{P})$ or $10 \%$ calf serum $(\mathrm{S})$ were used. The DNA-protein complexes were electrophoretically separated on $5 \%$ polyacrylamide gels, then dried and autoradiographed. The data are representative of five different experiments. 
a

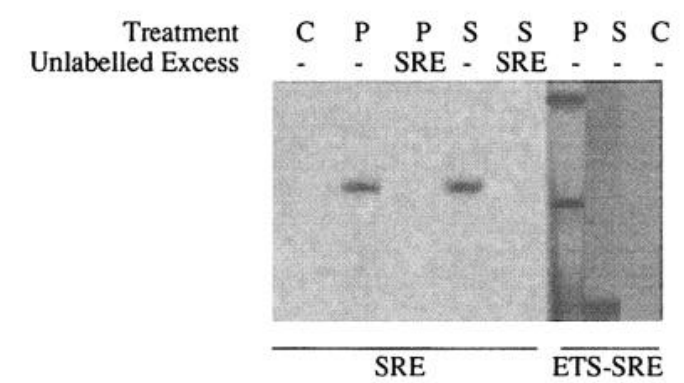

b

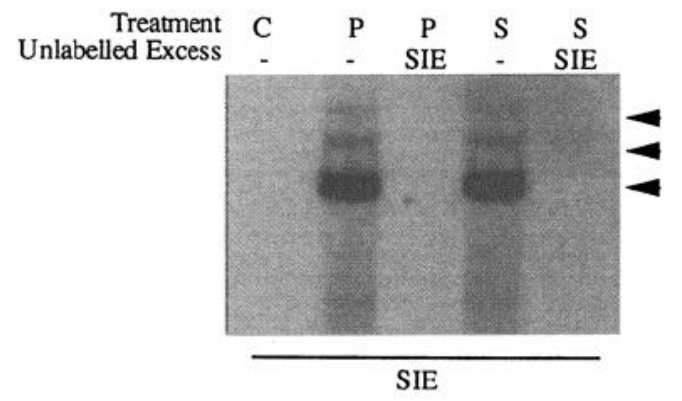

FIG. 4. Gel mobility shift assay with ${ }^{32}$ P-radiolabeled SRE and ETSSRE (a), and SIE (b) oligonucleotides. Nuclear extracts from Sertoli cells cultured in the absence (C) or presence of PModS(S300) (P) or $10 \%$ calf serum (S) were used. The DNA-protein complexes were electrophoretically separated on $5 \%$ polyacrylamide gels, then dried and autoradiographed. The data are representative of 10 different experiments for SRE and 5 different experiments for SIE.

upstream from the SRE, is the SIE, which is also responsive to serum. A SIE oligonucleotide gel shift demonstrated that PModS(S300) promotes a SIE gel shift (Fig. 4B). Again, serum was used as a positive control and promoted the anticipated gel shift. This SIE gel shift was specific and could be competed by excess unlabeled SIE oligonucleotide. Previously, a SIE gel shift has been shown to involve three shifts, termed $\mathrm{A}, \mathrm{B}$, and C. PModS was found to promote all three forms, with $\mathrm{A}$ (lowest) being the predominant (Fig. 4B). The gel shift data presented confirm the reporter gene experiments previously discussed. Therefore, PModS appears to act through the SRE and SIE to promote c-fos gene expression.

\section{Tf promoter analysis}

To determine the location of response elements within the Tf promoter that are activated by treatment with PModS, four CAT constructs containing a 581-bp, 1.6-kb, 2.6-kb, or $3-\mathrm{kb}$ upstream region of the Tf promoter were produced (Fig. $5 \mathrm{~A})$. FSH treatment of cells transfected with any of the constructs, except the 2.6- to 1.6-kb Tf PBL-CAT2, termed 1-kb Tf-Tk-CAT, stimulated CAT activity approximately $400 \%$ above control values, confirming that these constructs can be activated (data not shown; $P<0.01$ ). PModS(S300) treatment induced activation of the $-581-\mathrm{bp},-1.6-\mathrm{kb}$, and $-3-\mathrm{kb}$ Tf constructs (Fig. 5B) to a similar extent. The $-2.6-\mathrm{kb}$ Tf construct was stimulated to a greater extent by PModS(S300) (Fig. 5B). Interestingly, the PModS(C4) preparation stimu-

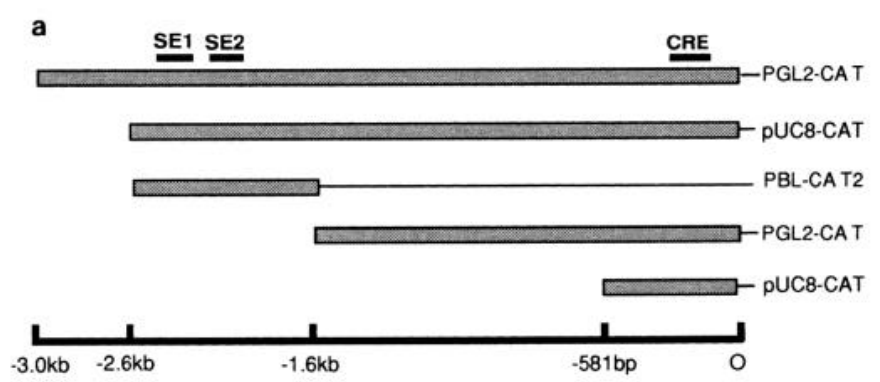

b

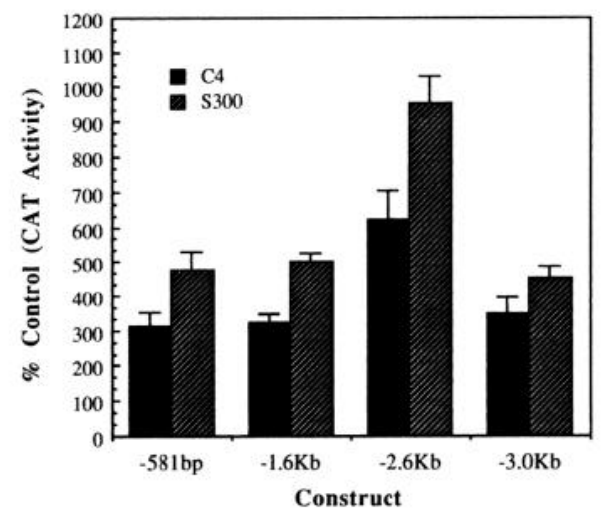

FIG. 5. a, Schematic representation of the Tf promotor-CAT constructs and location of CRE, SE1, and SE2 regions. b, Regulation of the various Tf promotor-CAT constructs in Sertoli cells cultured in the presence of PModS $(\mathrm{S} 300)(\mathbb{E})$ and $\mathrm{PModS}(\mathrm{C} 4)(\boldsymbol{E})$. Data are expressed as a percentage of the value in control nontreated cells for each construct. Data are representative of three different experiments performed twice.

lated primarily the $-2.6-\mathrm{kb}$ Tf construct. This indicates that an enhancer-like activity involved in PModS stimulation of Tf production is located between -1.6 and $-2.6 \mathrm{~kb}$, and a repressor is located between -2.6 and $-3 \mathrm{~kb}$ of the promoter. To extend these observations, the 1000 -bp region of the promoter between -1.6 and $-2.6 \mathrm{~kb}$ was cloned into a Tk-CAT construct (PBL-CAT2; Fig. 5A), termed $1 \mathrm{~kb}$ Tf-Tk-CAT. PModS was found to activate this construct, but had only a slight effect on the control plasmid not containing the 1-kb Tf promoter (Fig. 6). PModS(C4) had an action similar to that of the partially purified PModS(S300). The plasmid used contained a minimal thymidine kinase (Tk) promoter with a TATA box (Tk-CAT) that was required for PModS to activate this $1-\mathrm{kb}$ Tf construct. In the absence of a minimal promoter with a TATA box, a similar construct was not activated (data not shown). Therefore, the ability of PModS to activate the enhancer elements in this $\mathrm{Tf}$ promoter fragment does require a minimal promoter containing a TATA box.

Although the region between 0 and $-581 \mathrm{bp}$ has been sequenced and mapped, little is currently known about the distal region of the Tf promoter. Therefore, the exact nature of these putative upstream regulatory elements is unknown. To initiate an examination of this region, the entire $2.5-\mathrm{kb}$ fragment of the $\mathrm{Tf}$ promoter between $-581 \mathrm{bp}$ and $-3 \mathrm{~kb}$ was digested with various restriction enzymes to generate 15 approximately $200-\mathrm{bp}$ fragments. These fragments were used in gel mobility shift assays to identify potential PModS- 


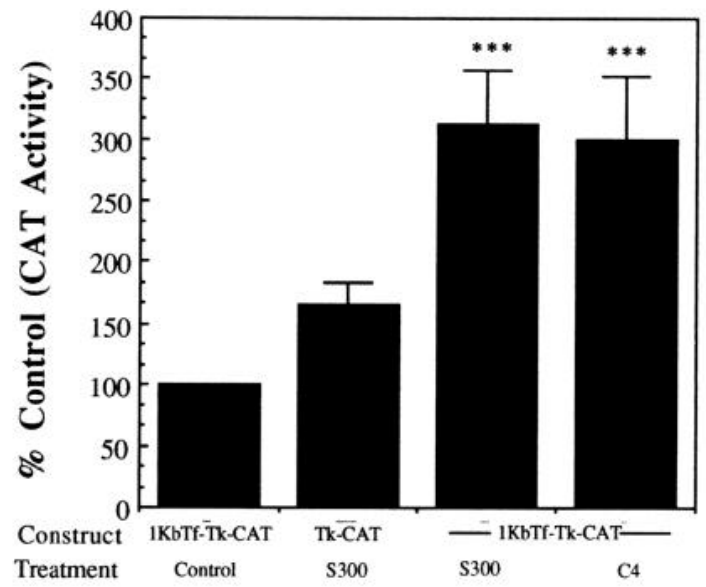

FIG. 6. Regulation of a 1-kb fragment of the Tf promoter between $-1.6 \mathrm{~kb}$ and $-2.6 \mathrm{~kb}$ containing SE1 and SE2 inserted into a Tk-CAT plasmid and termed $1 \mathrm{~kb}$ Tf-Tk-CAT. Sertoli cells were cultured in the absence (C) or presence of PModS(S300) or PModS(C4). The actions of PModS(S300) on a control Tk-CAT plasmid are also shown. Data are expressed as a percentage of the value in control nontreated cells for each construct and are presented as the mean \pm SEM from three different experiments performed twice. ${ }^{* * *}$, Statistically significant difference from the control $(P<0.01)$.

responsive regions. Thirteen of these $\mathrm{Tf}$ promoter restriction fragments did not cause a gel shift under control or PModSstimulated conditions (data not shown). Two restriction fragments upstream of the mTf transcriptional start site located at $-2.4 \mathrm{~kb}$, designated SE1, and at $-1.9 \mathrm{~kb}$, designated SE2, did cause a gel shift after PModS stimulation (Fig. 7). The location of these Sertoli elements (i.e. SE1 and SE2) on the Tf promoter is diagrammatically shown in Fig. 5A. To determine whether either SE1 or SE2 is a potential AP1-binding site, a competition experiment was carried out with excess unlabeled AP1 oligonucleotide. The unlabeled AP1 was not able to displace the SE1 or SE2 complex. Therefore, the gel shift appears not to be due to a c-fos/jun-binding AP1 site. The SE2 fragment was unable to form the DNA-protein complex with the nuclear extracts of Sertoli cells treated with serum (Fig. 7). PModS(S300) caused a gel shift with both SE1 and SE2 that was competed with excess unlabeled oligonucleotide (Fig. 7). Excess SE2 DNA fragment did not compete for the SE1 complex, nor did the SE1 compete for the SE2 complex. These two, apparently distinct, non-AP1-containing regions of the Tf promoter appear to be influenced by PModS actions on Sertoli cells.
To confirm that c-fos does not associate with the PModSinduced SE1 and SE2 gel shifts, an immunoblot with a c-fos antiserum of the gel shifts was performed (Fig. 8). Although a gel shift was detected with both SE1 and SE2, c-fos was not detected in the DNA-protein complex (Fig. 8). A positive control AP1 gel shift did contain c-fos in its DNA-protein complex (Fig. 8). These observations imply that c-fos regulates an intermediate transcription factor(s) that influences the Tf promoter at SE1 and SE2 (Fig. 9).

\section{Discussion}

The ability of the antisense c-fos oligonucleotide to inhibit PModS-induced activation of the Tf promoter in Sertoli cells transfected with the $-2.6-\mathrm{kb}$ Tf-CAT reporter construct correlates with its ability to inhibit $\mathrm{Tf}$ protein secretion (6). These data demonstrated that c-fos is involved in PModS stimulation of Tf expression by cultured Sertoli cells. To further investigate the actions of PModS on a molecular level, the effects of PModS on both the c-fos and Tf promoters were examined.

\section{c-Fos promoter analysis}

The responses of Sertoli cells treated with PModS and transfected with the reporter constructs containing the different regulatory elements of the c-fos promoter correlated with previous data obtained on the pharmacology of PModS. Treatment of cultured Sertoli cells with PModS did not affect cAMP levels (4), nor was it able to stimulate the reporter construct with the CRE of the c-fos promoter. The inability of PModS(S300) treatment to activate the TRE supports the observation that PModS does not stimulate either calcium mobilization or phosphoinositide turnover (5), both markers of protein kinase $\mathrm{C}$ activation. The inability of PModS to influence the TRE and AP1 (i.e. FAP) site at $-290 \mathrm{bp}$ of the c-fos promoter supports previous findings that PModS actions do not involve calcium mobilization or protein kinase $C$ activation (5). Only the SRE and SIE were stimulated by treatment with the PModS(S300) and the highly purified PModS(C4) preparations. Interestingly, the presence of both SRE and SIE was not additive; the effect was the same or lesser than that of either element alone. Therefore, the ability of PModS to induce c-fos expression appears to be through the activation of SRE and SIE.

The SRE is a 29-bp region within the c-fos promoter. A 67-kilodalton protein has been isolated that binds to the SRE, the SRF (18). Evidence indicates that the binding of SRF to
FIG. 7. Gel mobility shift assay with ${ }^{32}$ P-radiolabeled SE1 and SE2 regions of the $\mathrm{Tf}$ promoter. Nuclear extracts from Sertoli cells cultured in the absence (C) or presence of PModS(S300) $(\mathrm{P})$ or $10 \%$ calf serum $(\mathrm{S})$ were used. The DNA-protein complexes were electrophoretically separated on $5 \%$ polyacrylamide gels, then dried and autoradiographed. Incubations with excess unlabeled SE1, SE2, or AP1 are indicated. The data are representative of four different experiments.

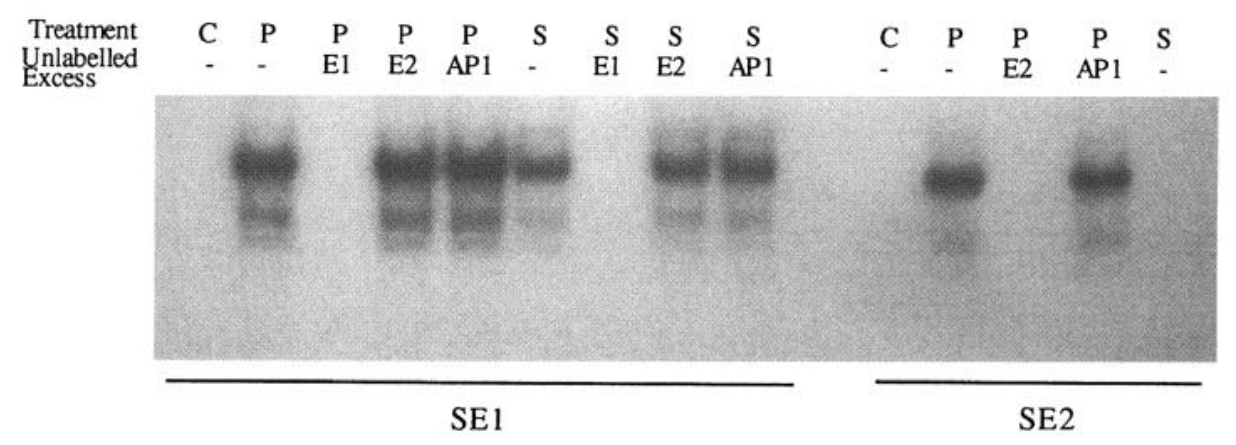




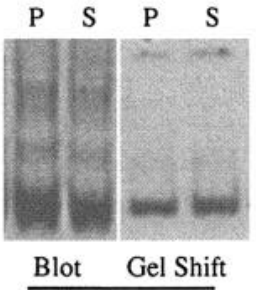

AP1

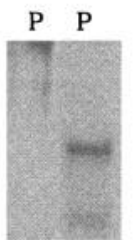

Blot Gel Shift

SE2

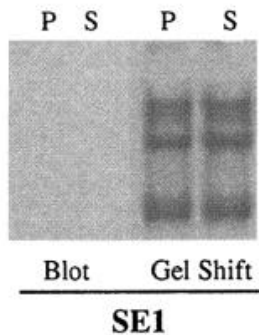

FIG. 8. Immunoblot of an AP1, SE1, and SE2 gel mobility shift assay with a c-fos antiserum. Nuclear extracts from Sertoli cells cultured in the presence of PModS (S300) (P) or 10\% calf serum (S) were used. The DNA-protein complexes were electrophoresed and separated on $5 \%$ polyacrylamide gels, then either dried and autoradiographed (Gel Shift) or electrophoretically blotted to polyvinylidene difluoride membrane and immunoblotted with the c-fos antiserum (Blot). The data are representative of two experiments.

SRE requires its phosphorylation (19-21). The actions of PModS require tyrosine phosphorylation events (5). The DNA-binding domain of SRF is the region necessary for dimerization and interaction with other proteins to form ternary complexes (18). Because SRF is ubiquitously expressed, cell-specific activation of the c-fos SRE by SRF appears to be mediated by other ternary complex protein factors. The binding of the ternary complex to the SRE requires the presence of the adjoining 5 '-side of the SRE-binding site, the ETS domain binding motif. The protein kinase $\mathrm{C}$-dependent and -independent pathways target different parts of this ternary complex (22). The expression and/or phosphorylation of these proteins may be involved in mediating cellspecific responses by the SRE. The SRE gel shift data presented demonstrate that PModS induced a gel shift similar to that of the positive control with serum treatment. Under less stringent conditions, a SRE gel shift was detected in control cells, but a more abundant gel shift was present in PModS-treated Sertoli cells (data not shown). The identity of the PModS-induced SRE-binding protein as SRF or other binding proteins remains to be elucidated. Interestingly, PModS induced a unique gel shift with the ETS-SRE that was not observed in control nontreated cells or in serum-treated cells. Therefore, PModS appears to promote the binding of a protein complex (e.g. ternary complex) that activates the SRE within the c-fos promoter. The formation of these ternary complexes may enable a common regulatory element, such as the SRE, to have cell-specific activity using ubiquitously expressed DNA-binding proteins. Alternatively, different or unique binding factors may be another method by which a common regulatory element may differentially activate cellspecific gene expression. In support of this proposal, several other DNA-binding factors have been identified that interact with the SRE. The 62-kilodalton SRE direct binding factor $\left(\mathrm{p} 62^{\mathrm{DBF}}\right.$ ) may be related to the MAPF1 protein isolated from yeast (23). This factor is phosphorylated in vivo and mediates muscle-specific expression of $\alpha$-actin. SRE-zinc-binding protein is a member of the $\mathrm{C}_{2} \mathrm{H}_{2}$ zinc finger family (24). High affinity binding is achieved through seven tandemly repeated zinc finger motifs. Rat NFIL-6 is a C/EBP-related factor whose binding to the SRE is mutually exclusive with SRF (25). Further analysis of activation of the SRE by PModS will investigate the potential involvement of known ternary complex factors, unique ternary complex factors, or novel SRE-binding proteins.

A second regulatory element that appears to be involved in PModS stimulation of c-fos is the SIE. It is located $25 \mathrm{bp}$ upstream of the SRE. The SIE is activated by binding of the SIF, which has three forms, A, B, and C, and contains a phosphotyrosine residue (11). Treatment of human epidermoid carcinoma cells with epidermal growth factor and human hepatoma cells with interleukin- 6 induces the binding of SIF-A, whereas treatment of epidermoid cells with interferon- $\gamma$ induced binding of SIF-C (11). This is also an example of the ability of different binding proteins to activate a common regulatory element and stimulate a gene-specific response. PModS induced a SIE gel shift that appears to involve predominantly $\operatorname{SIF}(\mathrm{A})$, but also had detectable $\operatorname{SIF}(\mathrm{B})$ and SIF(C) gel shifts. PModS was also found to activate a SIE reporter construct. Interestingly, the effects of both the SIE and SRE were not additive in response to PModS. PModS appears to influence the c-fos promoter at both the SRE and SIE. Whether the SIE can compensate and/or regulate the SRE response remains to be elucidated.

\section{Tf promoter analysis}

The downstream effects of PModS-induced c-fos expression were investigated with use of the Tf gene as a differentiated marker of Sertoli cells. The gene expression of $\mathrm{Tf}$ was inhibited by an antisense c-fos oligonucleotide, indicating that actions of PModS on the Tf promoter are indeed a downstream c-fos event. The regions controlling liver-specific transcription of the human Tf gene are composed of multiple positive and negative acting elements, mostly interacting with DNA-binding proteins present in either human or rat liver nuclear extracts $(26,27)$. The regulation of $\mathrm{Tf}$ gene expression in unstimulated Sertoli cells has been shown to be distinct from that of liver gene expression (28). Although the first $581 \mathrm{bp}$ of the human Tf promoter (hTf) and mTf promoter have little similarity, they both contain a CRE site,
FIG. 9. Schematic of the proposed molecular actions of PModS to regulate Sertoli cell differentiation.
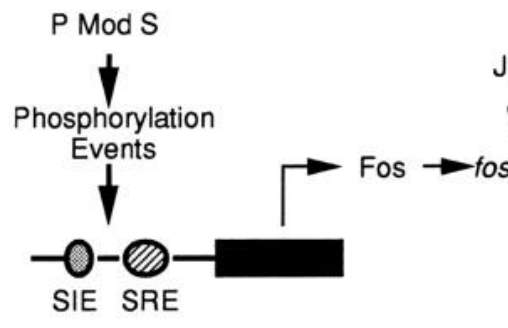
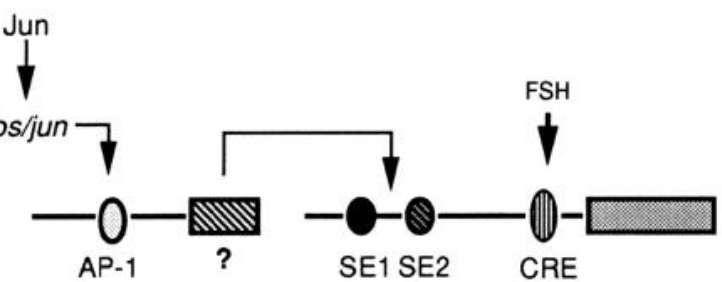
which was found to be responsive to FSH in the current study. The results with the CAT-mTf deletion mutants suggest that an enhancer activity is located between -2.6 and $-1.6 \mathrm{~kb}$ for PModS and a repressor between -2.6 and -3.0 $\mathrm{kb}$. Analysis of restriction enzyme fragments of the Tf promoter between -581 bp and $-3 \mathrm{~kb}$ identified two distinct domains, designated SE1 and SE2, that bind specific nuclear proteins from Sertoli cells stimulated with PModS. Interestingly, these Sertoli response elements (i.e. SE1 and SE2) are locatcd within the apparent enhancer activity between -2.6 and $-1.6 \mathrm{~kb}$ of the Tf promoter. Treatment with serum also induced a similar DNA-protein complex with SE1. The complex formed with SE2 was specific to PModS(S300), because treatment with serum or cAMP (data not shown) could not induce the gel shift. The lack of competition of SE1 and SE2 with AP1 suggested that these sites were independent of AP1 and the binding of a c-fos-jun complex. The c-fos immunoblot confirmed the absence of c-fos in the SE1 and SE2 DNAprotein complexes. Therefore, PModS induced Tf gene expression may involve both c-fos-dependent and -independent response elements. It is postulated that $c$-fos regulates the expression or activities of an intermediate transcription factor(s) that subsequently binds the SE1 and SE2 regions. The general mechanism is currently proposed, in that PModS, through activation of the SRE and SIE, induces the immediate carly response gene c-fos, which then influences an intermediate transcription factor(s) that regulates downstream Sertoli cell differentiated functions such as Tf gene expression. Future studies will involve elucidation of the precise mechanism by which PModS activates the SRE and SIE of the c-fos promoter and Sertoli cell-specific response elements (e.g.SE1 and SE2) of the Tf promoter. The possibility that Sertoli cell-specific trans-acting factors and ternary complex factors as well as unique response elements may be involved in the Sertoli cell-specific activation of these promoters is being investigated.

\section{Acknowledgments}

We thank Lisa Halburnt and Betty Chu for technical assistance and Jennifer Simenc and Pamela Garay-Kobuchi for assistance with the preparation of the manuscript. We also thank Dr. Jean-Louis Vigne for valuable assistance.

\section{References}

1. Skinner MK, Fritz IB 1985 Testicular cells secrete a protein under androgen control that modulates Sertoli cell function. Proc Natl Acad Sci USA 82:114-118

2. Skinner MK, Fetterolf PM, Anthony CT 1988 Purification of the paracrine factor, P-Mod-S, produced by testicular peritubular cells that modulates Sertoli cell function. J Biol Chem 263:2884-2890

3. Skinner MK, Griswold MD 1980 Sertoli cells synthesize and secrete a transferrin-like protein. J Biol Chem 255:9523-9525

4. Norton JN, Skinner MK 1989 Regulation of Sertoli cell function and differentiation through the actions of a testicular paracrine factor PModS. Endocrinology 124:2711-2719

5. Norton JN, Vigne J-L, Skinner MK 1994 Regulation of Sertoli cell differentiation by the testicular paracrine factor PModS: analysis of common signal transduction pathways. Endocrinology 134:149-157

6. Norton JN, Skinner MK 1992 Regulation of Sertoli cell differentiation by the testicular paracrine factor PModS: potential role of immediate-early genes. Mol Endocrinol 6:2018-2026
7. Deschamps J, Meijlink F, Verma IM 1985 Identification of transcriptional enhancer element upstream from the proto-oncogene cfos. Science 230:1174-1177

8. Dorrington JH, Roller NF, Fritz IB 1975 Effects of follicle-stimulating hormone on cultures of Sertoli cell preparations. Mol Cell Endocrinol 3:57-70

9. Tung PS, Skinner MK, Fritz IB 1984 Fibronectin synthesis is a marker for peritubular cell contaminants in Sertoli cell enriched cultures. Biol Reprod 30:199-211

10. Luckow B, Schutz G 1987 CAT constructions with multiple unique restriction sites for the functional analysis of eukaryotic promoters and regulatory elements. Nucleic Acids Res 15:5490

11. Sadowski HB, Shuai K, Darnell JE, Gilman MZ 1993 A common nuclear signal transduction pathway activated by growth factor and cytokine receptors. Science 261:1739-1744

12. Idzerda RL, Behringer RR, Theisen M, Huggenick JI, McKnight GS, Brinster RL 1989 Expression from the transferrin gene promoter in transgenic mice. Mol Cell Biol 9:5154-5162

13. Kingston RE, Chen CA, Okayama H 1993 Calcium phosphate transfection. In: Ausabel FA, Brent R, Kingston RE, Moore DD, Seidman JG, Smith, JA, Struhl K (eds) Current Protocols in Molecular Biology. Greene and Wiley Interscience, New York, pp 9.1.1-9.1.3

14. Guillou F, Zakin MM Part D, Boissier Schaeffer E 1991 Sertoli cell specific expression of the human transferrin gene. J Biol Chem 266: 9876-9884

15. Garner MM, Rezvin A 1981 A gel electrophoresis method for quantifying the binding of proteins to specific DNA regions: application to components of the Escherichia coli lactose operon regulatory system. Nucleic Acids Res 9:3047-3059

16. Holt JT, Redner RL, Nienhuis AW 1988 An oligomer complementary to c-myc mRNA inhibits proliferation of HL-60 promyelocytic cells and induced differentiation. Mol Cell Biol 8:963-973

17. Kerr LK, Holt JT, Matrisian LM 1988 Growth factors regulate transin gene expression by cfos-dependent and cfos-independent pathways. Science 242:1424-1427

18. Schroter H, Mueller CGF, Meese K, Nordheim A 1990 Synergism in ternary complex formation between the dimeric glycoprotein p $67^{\mathrm{SRF}}$, polypeptide $\mathrm{p} 62^{\mathrm{TCF}}$ and the cfos serum response element. EMBO J 9:1123-1130

19. Prywes R, Dutta A, Cromlish JA, Roeder RG 1988 Phosphorylation of serum response factor, a factor that binds to the serum response element of the cfos enhancer. Proc Natl Acad Sci USA 85:7206-7210

20. Triesman R 1992 The serum response element. TIBS 17:423-426

21. Gauthier-Rouviere C, Basset M, Blanchard J-M, Cavadore J-C, Fernandez A, Lamb NJC 1991 Casein kinase II induces c-fos expression via the serum response element pathway and $\mathrm{p} 67^{\mathrm{SRF}}$ phosphorylation in living fibroblasts. EMBO f 10:2921-2930

22. Graham R, Gilman M 1991 Distinct protein targets for signals acting at the cfos serum response element. Science 251:189-9223

23. Ryan WA, Franza BR, Gilman MZ 1989 Two distinct cellular phosphoproteins bind to the c-fos serum response element. EMBO J 8:1785-1792

24. Attar RM, Gilman MZ 1992 Expression cloning of a novel zinc finger protein that binds to the cfos serum response element. Mol Cell Biol $12: 2432-2443$

25. Metz R, Ziff E 1991 The helix-loop-helix protein $\mathrm{rE} 12$ and the C/EBP-related factor rNFIL- 6 bind to neighboring sites within the cfos serum response element. Oncogene 6:2165-2178

26. Brunel F, Ochoa A, Schaeffer E, Boisser F, Guillou Y, Cereghini S, Cohen GN, Zakin MM 1988 Interactions of the DNA binding proteins with the $5^{\prime}$ region of the human transferrin gene. J Biol Chem 263:10180-10185

27. Schaeffer E, Boissier F, Py MC, Cohen GN, Zakin MM 1989 Cell specific expression of the human transferrin gene. J Biol Chem 264: $7153-716028$

28. Schaffer E, Guillou F, Part D, Zakin M 1993 A different combination of transcription factors modulates the expression of the human transferrin promoter in liver and Sertoli cells. J Biol Chem 268: $23399-23408$ 